\title{
PRODUCCIÓN EDITORIAL DE LAS UNIVERSIDADES CHILENAS ENTRE 2002 Y 2004
}

Manuel Loyola* 



\section{PRODUCCIÓN EDITORIAL DE LAS UNIVERSIDADES CHILENAS ENTRE 2002 Y 2004}

Si bien todas las universidades chilenas cuentan con algún tipo de producción editorial (cualquiera sea el formato o soporte de presentación), la inserción de esta producción en registros de orden internacionales (ISSN e ISBN) representa un muy claro indicador del interés o relevancia que para estas instituciones tiene esta actividad, de manera que, sin temor a equivocarnos, la constatación de códigos de este orden en los productos editados brinda al lector o consultante una entrada que gana su confianza.

En el presente informe se da a conocer una visión de la producción editorial de las universidades del país para el periodo 2002 y 2004. La base cuantitativa de esta visión corresponde únicamente a la producción editorial universitaria que cuenta con registro ISBN (International Standard Book Number), por tanto, los datos pertenecen a productos monográficos (libros, textos de estudio, CD's, DVD's, videos, entre otros), es decir, de título independiente o que no son parte de ninguna edición seriada, como revistas, apuntes o documentos que realizan las unidades editoras universitarias $^{1}$.

\section{Registros ISBN de las universidades chilenas: visión de contexto}

La producción universitaria con ISBN entre los años 2002 y 2004 ha representado, en promedio, el 10\% de los títulos registrados en

1 De acuerdo con la normativa vigente (Ley 19.227), los productos de título independiente, junto con su inscripción de propiedad intelectual, deben disponer de su respectivo registro ISBN, método de clasificación de la producción editorial de enorme valor para el mundo del libro. Este sistema es administrado en Chile por la Cámara Chilena del Libro. 
Chile, acusando un incremento porcentual similar al aumento anual de títulos provenientes de los sectores no universitarios ${ }^{2}$ (como se aprecia en la tabla $\mathrm{N}^{\circ} 1$ ).

Tabla $N^{\circ} 1$. Registros ISBN nacionales. 2002-2004

\begin{tabular}{|c|c|c|c|c|c|}
\hline Años & $\begin{array}{c}\text { Producción no } \\
\text { universitaria }\end{array}$ & $\begin{array}{c}\text { Producción } \\
\text { universitaria }\end{array}$ & $\begin{array}{c}\% \text { no } \\
\text { universitaria }\end{array}$ & $\begin{array}{c}\% \\
\text { universitaria }\end{array}$ & Totales \\
\hline 2002 & 2.835 & 307 & 90,1 & 9,9 & 3.142 \\
\hline 2003 & 3.420 & 371 & 90,2 & 9,8 & 3.791 \\
\hline 2004 & 3.151 & 375 & 89,4 & 10,6 & 3.526 \\
\hline Totales & 9.406 & 1.053 & 89,9 & 10,1 & 10.459 \\
\hline
\end{tabular}

Deteniéndonos en la revisión de la producción netamente universitaria, los datos arrojan una relación altamente asimétrica entre la producción editada por las universidades del Consejo de Rectores y la realizada por las universidades privadas (ver tabla $\mathrm{N}^{\circ} 2$ ). Obviamente, esto se explica por razones de antigüedad y complejidad académicas presentes en las del Consejo, además de la mayor capacidad de recursos humanos y financieros disponibles por estas mismas universidades respecto de las privadas.

Tabla No 2. Registros ISBN sectorial. Universidades privadas y del Consejo de Rectores. 2002-2004

\begin{tabular}{|c|c|c|c|c|}
\hline Años & $\begin{array}{c}\text { Universidades } \\
\text { privadas }\end{array}$ & $\begin{array}{c}\text { Universidades } \\
\text { C. de Rectores }\end{array}$ & \% privadas & \% C. de Rectores \\
\hline 2002 & 66 & 241 & 21,5 & 78,5 \\
\hline 2003 & 70 & 301 & 18,9 & 81,1 \\
\hline 2004 & 73 & 302 & 19,5 & 80,5 \\
\hline Totales & 209 & 844 & 19,8 & 80,2 \\
\hline
\end{tabular}

Si bien, en promedio, los registros ISBN en el sector privado de la educación universitaria representan prácticamente el 20\% de la producción del sistema en su conjunto, el comportamiento de este ámbito en los años consultados verifica un crecimiento real muy leve, con una pequeña pérdida en la magnitud porcentual. La

2 No disponemos, por el momento, de informaciones comparativas o estimativas que nos permitan señalar si esta proporción de un décimo de la producción nacional es adecuada o no para las características de producción y consumo de productos editoriales de nuestro país. 
producción con registro ISBN de las universidades del Consejo de Rectores, por su parte, experimenta un aumento, en especial por el alza experimentada en 2003 respecto del año anterior, la que se mantiene en 2004.

La identificación por universidad de los registros ISBN nos informa del grado de concentración que existe en materia de producción editorial. En efecto, del total de 41 universidades (ver antecedentes completos en la tabla A, al final del texto), el 24,3\% de ellas totalizaron el 71,6\% de los registros ISBN inscritos entre 2002 y 2004 (ver tabla No 3).

Tabla $N^{0}$ 3. Universidades con mayor cantidad de registros ISBN

\begin{tabular}{|l|c|}
\hline Institución & $\begin{array}{c}\text { Total de registros ISBN } \\
\text { 2002-2004 }\end{array}$ \\
\hline Pontificia Universidad Católica de Chile & 157 \\
\hline Universidad Católica del Norte & 101 \\
\hline Universidad de Chile & 95 \\
\hline Universidad Arturo Prat & 86 \\
\hline Universidad de Playa Ancha & 74 \\
\hline Universidad de Concepción & 68 \\
\hline Universidad de Arte y Ciencias Sociales (ARCIS) & 47 \\
\hline Universidad Diego Portales & 47 \\
\hline Pontificia Universidad Católica de Valparaíso & 41 \\
\hline Universidad de Antofagasta & 38 \\
\hline Total registros 10 universidades & 754 \\
\hline Total registros 4l universidades & 1.053 \\
\hline
\end{tabular}

No obstante, esta concentración adquiere más relevancia al tomar en cuenta sólo las cinco primeras universidades de la tabla $\mathrm{N}^{\circ} 3$, que alcanzaron casi el 50\% del total de los registros (513). Finalmente, un par de aspectos significativos de este ranking: la destacada posición de las ediciones de la Pontificia Universidad Católica de Chile, con un 15\% del total, y la presencia de dos universidades privadas - de Arte y Ciencias Sociales (ARCIS) y Diego Portales- entre las de mayores registros, tema al cual nos referiremos enseguida. 


\section{Ediciones con ISBN en las universidades privadas}

Como fue mencionado, la proporción de registros ISBN en el sistema universitario es mayor en las ediciones de las universidades del Consejo de Rectores. Desde un punto de vista general, las publicaciones de las privadas representan el $2 \%$ del total de los registros en el ámbito nacional inscritos entre 2002 y 2004. No obstante, un par de universidades privadas logran colocarse entre las unidades editoras con mayores cantidades de registros, pero, en ningún caso pueden ser medidas en el ámbito de las universidades del Consejo. Lo más adecuado es que se comparen con sus similares del sector privado, con el fin de dimensionar de mejor forma sus esfuerzos.

La tabla $\mathrm{N}^{\circ} 4$ proporciona los datos generales de la realidad editorial ISBN de las universidades privadas para los últimos tres años.

Tabla No 4. Registros ISBN de las universidades privadas. 2002-2004

\begin{tabular}{|l|c|c|}
\hline Institución & $\begin{array}{c}\text { Total de } \\
\text { registros ISBN } \\
\text { 2002-2004 }\end{array}$ & $\begin{array}{c}\text { \% del total de } \\
\text { universidades } \\
\text { privadas }\end{array}$ \\
\hline Universidad de Arte y Ciencias Sociales (ARCIS) & 47 & 22,5 \\
\hline Universidad Diego Portales & 47 & 22,5 \\
\hline Universidad Católica Silva Henríquez & 28 & 13,4 \\
\hline Universidad Bolivariana & 13 & 6,2 \\
\hline Universidad de los Andes & 10 & 4,8 \\
\hline Universidad Andrés Bello & 10 & 4,8 \\
\hline Universidad Gabriela Mistral & 7 & 3,3 \\
\hline Universidad Mayor & 7 & 3,3 \\
\hline Universidad Academia de Humanismo Cristiano & 6 & 2,9 \\
\hline Universidad José Santos Ossa & 6 & 2,9 \\
\hline Universidad Santo Tomás & 5 & 2,4 \\
\hline Universidad de Viña del Mar & 5 & 2,4 \\
\hline Universidad del Desarrollo & 4 & 1,9 \\
\hline Universidad San Sebastián & 3 & 1,4 \\
\hline Universidad Pérez Rosales & 3 & 1,4 \\
\hline Universidad Adolfo Ibáñez & 2 & 1,0 \\
\hline Universidad La República & 2 & 1,0 \\
\hline Universidad Alberto Hurtado & 1 & 0,5 \\
\hline Universidad Iberoamericana de Ciencias y Tecnología. & 1 & 0,5 \\
\hline Universidad de Ciencias de la Informática & 1 & 0,5 \\
\hline Universidad Mariano Egaña & 1 & 0,5 \\
\hline Total & 209 & 100,0 \\
\hline
\end{tabular}


La polarización de registros es un hecho destacable de los datos anotados: mientras las tres primeras universidades privadas — $14,2 \%$ de éstas— reportan el 58,4\% de los registros, las últimas cuatro (19\%) sólo aportan el 2\% del total. Aún más, si establecemos que el promedio del sector es de 9,5 registros para los tres años consultados ( 3,2 por año), tendremos que 15 de estas universidades están por debajo de esta ponderación, lo que equivale al 71,4\% del conjunto de las universidades privadas ${ }^{3}$.

Ahora bien, para un análisis más específico del comportamiento editorial desde los registros hechos por estas instituciones privadas, hemos establecido una nomenclatura clasificatoria ad hoc dada por los siguientes términos:

- Alto Nivel de Registros (ANR) para una cantidad igual o superior a diez registros anuales.

- Mediano Nivel de Registros (MNR) para una cantidad igual o superior a cinco registros anuales.

- Bajo Nivel de Registros (BNR) para una cantidad igual o inferior a los cuatro registros anuales.

3 En el caso de las instituciones del Consejo de Rectores, el promedio para los años en estudio es de 42,2 registros, o 14 al año. No alcanzan este promedio 12 universidades de este sector, lo que equivale al $60 \%$ de ellas. 


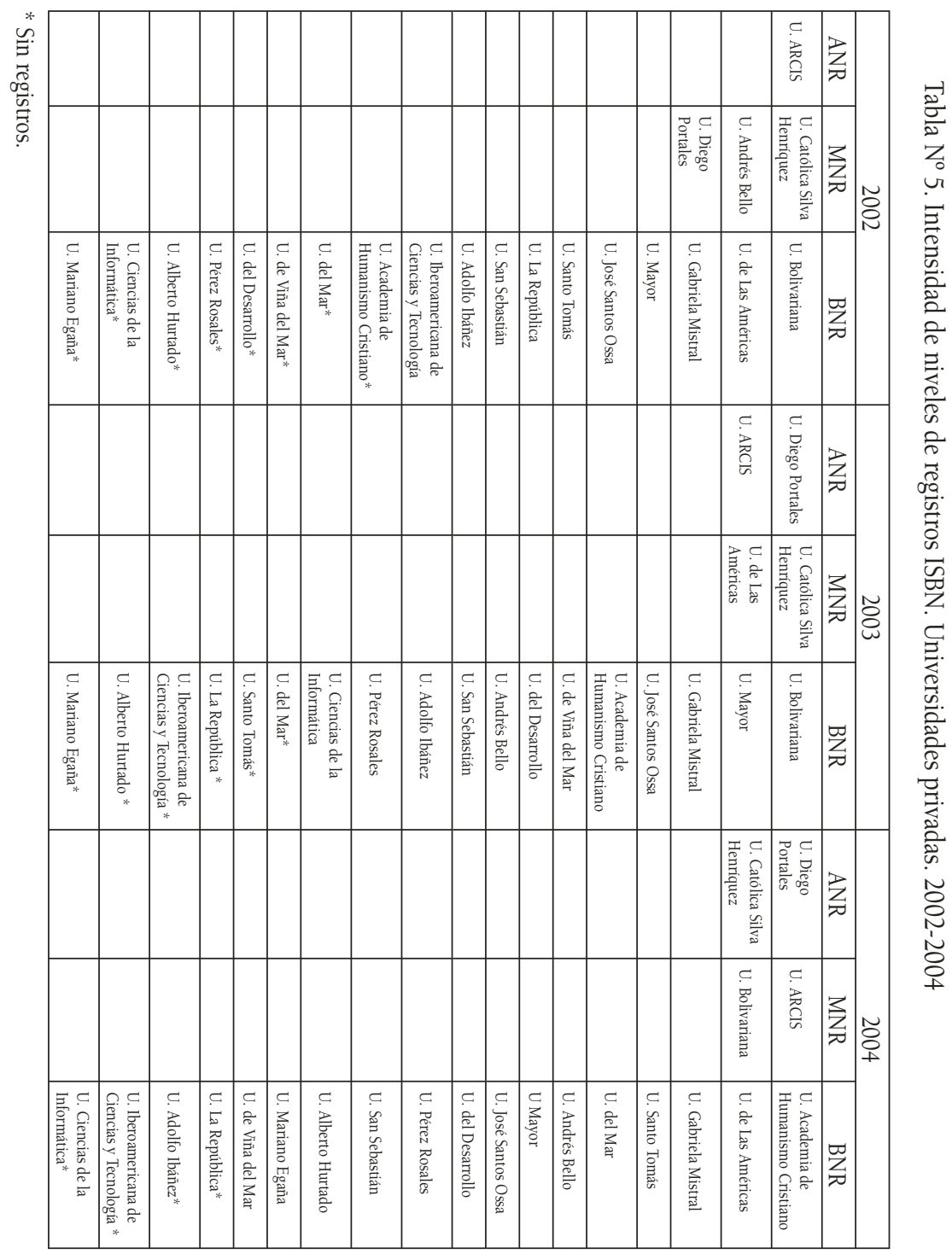

Mencionemos algunos alcances derivados de la tabla precedente.

a. Es clara la tendencia de estas universidades a la incorporación, aunque lenta, a las ediciones con registro ISBN. De ocho 
universidades que en 2002 no señalan registros, se pasa a cuatro sin registros en 2004. Persisten situaciones poco homogéneas o vacilantes en este proceso por parte de algunas universidades, como es el caso de las universidades Del Mar, Mariano Egaña, La República e Iberoamericana de Ciencias y Tecnología.

b. Mayoritariamente, las universidades privadas se incorporan y se mantienen en un nivel bajo de registros; no obstante, no son pocos los casos en que su manutención en este nivel resulta difícil, cayendo a lugares menores dentro del mismo, o saliendo de él: situación de las universidades La República, Adolfo Ibáñez e Iberoamericana de Ciencias y Tecnología. Una actuación destacada en este nivel le cabe a la Universidad Bolivariana, que, manteniéndose en los primeros lugares de este rango en 2002 y 2003, sube al nivel medio en 2004.

c. Entre las que reportan nivel medio de registros, se produce un estancamiento en su baja cantidad (no más de tres, en el mejor de los casos, en 2002). Aquí el comportamiento deficiente corrió por cuenta de la Universidad Nacional Andrés Bello, que en 2003 cae al nivel bajo.

d. Por su parte, la Universidad Diego Portales demuestra un fuerte dinamismo en el transcurso de los años en revisión, alcanzando el tramo de nivel alto en 2003 y manteniendo el mismo nivel al año siguiente. Con un dinamismo similar, aunque menor respecto de la institución anterior, la Universidad Católica Silva Henríquez también ofrece un ritmo ascendente en sus niveles de registros, accediendo a la categoría principal en 2004.

e. Entre los cambios más evidentes expuestos por las universidades privadas que lideran en los registros ISBN, la situación de ARCIS es la más notoria, por la espectacularidad de su caída: de 29 títulos registrados en 2002, llega a ocho en 2004; pasando del nivel alto al medio, compartiendo la posición con la Universidad Bolivariana, que en 2004 también registró ocho títulos. 


\section{Registros ISBN universitarios por materia}

Finalizaremos esta visión dando a conocer la distribución por materias en las ediciones con registro ISBN de las universidades privadas y del Consejo de Rectores. La clasificación de materias corresponde a la que utiliza la Cámara Chilena del Libro (ver tabla № 6).

Tabla $N^{\circ} 6$. Ediciones por materias. Total universidades, 2002-2004

\begin{tabular}{|l|c|c|}
\hline Materia & $\begin{array}{c}\text { Número de ediciones de } \\
\text { universidades privadas }\end{array}$ & $\begin{array}{c}\text { Número de ediciones de } \\
\text { universidades del Consejo } \\
\text { de Rectores }\end{array}$ \\
\hline Filosofía y pensamiento & 21 & 36 \\
\hline Religión & 1 & 13 \\
\hline Ciencias Sociales & 43 & 91 \\
\hline Economía & 9 & 31 \\
\hline Derecho & 18 & 25 \\
\hline Administración & 9 & 36 \\
\hline Educación & 40 & 146 \\
\hline Folclore & 0 & 12 \\
\hline Lenguas & 0 & 141 \\
\hline Ciencias Puras & 4 & 68 \\
\hline Tecnología & 10 & 33 \\
\hline Artes-Recreación & 7 & 98 \\
\hline Literatura & 20 & 89 \\
\hline Historia y Ciencias Auxiliares & 23 & 17 \\
\hline Generalidades & 4 & 844 \\
\hline Total & 209 & \\
\hline
\end{tabular}

En las ediciones con registro ISBN de las universidades privadas predominan abiertamente las ligadas a las áreas de Educación, Ciencias Sociales y Humanidades. Esto queda más claro si allegamos a las dos últimas las cifras de las ediciones de Economía, Administración, Literatura, Derecho e Historia. En total ellas alcanzan la cantidad de 201 publicaciones, lo que representa el 96,1 del conjunto. Obviamente, los sectores de Ciencias Puras, Folclore, Lenguas y Religión poseen una muy menor relevancia.

Por su lado, las ediciones en Educación, Ciencias Sociales y Humanidades (a las que sumamos también las de Derecho, 
Economía, Administración, Literatura e Historia) provenientes de las universidades del Consejo, representan el 69,3\% del total de registros (585 de 844), consignándose, por tanto, una participación más nítida —en comparación con las universidades privadas- de las relativas a Ciencias Puras (16,7\% del total), Folclore o Lenguas.

Si tenemos en cuenta que los registros del Consejo de Rectores cuadruplican a los de las universidades privadas, podemos hacer una comparación considerando esta proporción, es decir, indicar en qué materias las ediciones de las privadas mantienen o no la brecha. Para esto, dividimos los registros del Consejo por los de las privadas y, donde el resultado sea igual o menor a cuatro, estaremos en presencia de una relación similar. En caso contrario, la relación será deficitaria o negativa (ver tabla $\mathrm{N}^{\circ} 7$ ).

Tabla $N^{0} 7$. Relación de proporcionalidad de universidades privadas respecto de las del Consejo de Rectores

\begin{tabular}{|l|c|c|c|}
\hline Materia & $\begin{array}{c}\text { Número de ediciones de } \\
\text { universidades privadas }\end{array}$ & $\begin{array}{c}\text { Número de ediciones } \\
\text { de universidades del } \\
\text { Consejo de Rectores }\end{array}$ & Relación \\
\hline Filosofía y pensamiento & 21 & 36 & 1,7 \\
\hline Religión & 1 & 13 & 13 \\
\hline Ciencias Sociales & 43 & 91 & 2,1 \\
\hline Economía & 9 & 31 & 3,4 \\
\hline Derecho & 18 & 25 & 1,3 \\
\hline Administración & 9 & 36 & 4,0 \\
\hline Educación & 40 & 146 & 3,6 \\
\hline Folclore & 0 & 8 & 8 \\
\hline Lenguas & 0 & 12 & 12 \\
\hline Ciencias Puras & 10 & 68 & 35,2 \\
\hline Tecnología & 7 & 33 & 6,8 \\
\hline Artes-Recreación & 20 & 98 & 4,7 \\
\hline Literatura & 23 & 89 & 3,9 \\
\hline $\begin{array}{l}\text { Historia y Ciencias } \\
\text { Auxiliares }\end{array}$ & 209 & 17 & 4,2 \\
\hline Generalidades & & 844 & 4,0 \\
\hline Total & 4 & & \\
\hline
\end{tabular}


Ordenando de modo decreciente las proporcionalidades, la situación para las privadas se presenta de la siguiente manera (ver tabla $\mathrm{N}^{\circ} 8$ ).

Tabla $\mathrm{N}^{\circ}$ 8. Orden decreciente de proporcionalidades. Universidades privadas con relación a universidades del Consejo de Rectores

\begin{tabular}{|l|c|}
\hline Materia & Relación \\
\hline Derecho & 1,3 \\
\hline Filosofía y pensamiento & 1,7 \\
\hline Ciencias Sociales & 2,1 \\
\hline Economía & 3,4 \\
\hline Educación & 3,6 \\
\hline Historia y Ciencias Auxiliares & 3,9 \\
\hline Administración & 4,0 \\
\hline Generalidades & 4,2 \\
\hline Artes-Recreación & 4,7 \\
\hline Literatura & 4,9 \\
\hline Tecnología & 6,8 \\
\hline Folclore & 8,0 \\
\hline Lenguas & 12,0 \\
\hline Religión & 13,0 \\
\hline Ciencias Puras & 35,2 \\
\hline
\end{tabular}

Siendo las ediciones ligadas a Ciencias Sociales, Educación y Humanidades las de mayor producción por parte del ámbito privado, resulta lógico que éstas demuestren una adecuada proporcionalidad respecto de los registros del Consejo, al menos hasta la materia de Administración. Seguidamente, esta proporcionalidad se torna crecientemente negativa, en tanto las materias objeto de ediciones implican insumos cada vez más complejos o especializados, que demandan recursos en investigación y sistematización, y que difícilmente logran ser asumidos por las privadas. Mientras las áreas de edición en las privadas corresponden preferentemente a disciplinas "blandas", las editadas por las universidades del Consejo poseen mayor equilibrio entre "blandas y "duras" (no olvidemos que en las universidades de Chile, Católica y de Concepción se concentra buena parte de la investigación universitaria en Chile). 


\section{Conclusiones}

Las ediciones universitarias en Chile con registro ISBN son una cifra menor dentro del conjunto de ediciones registradas en el plano nacional (sólo el 10\%). De esta décima parte, los registros de las universidades del Consejo de Rectores cuadriplican a los de las universidades privadas ( $8 \%$ y $2 \%$, respectivamente).

En ambos sectores se produce un fenómeno de concentración de registros en pocas universidades, de manera que existe una mayoría de casas de estudio superiores (cerca del 70\%) que, o producen muy poco, o no se han preocupado por formalizar su producción en registros internacionales (ISBN, ISSN), cuestión que además se condice con el escaso desarrollo de la gestión editorial propia y sectorial.

A diferencia de las universidades del Consejo de Rectores, las ediciones ISBN de las universidades privadas arrojan un desequilibrio o polarización temática, en especial, respecto de las disciplinas "blandas" o de baja complejidad. No obstante, debemos reconocer también el fenómeno de concentración en ediciones de materias "duras" que acusan las ediciones del Consejo (en no más de cuatro universidades).

Las ediciones de la Universidad Católica Silva Henríquez, respondiendo a una trayectoria, han logrado colocarse entre las más dinámicas del sector universitario privado y, comparativamente, sobrepasan incluso a varias universidades del Consejo de Rectores.

A fin de tener una mirada más acabada del tema editorial universitario, será preciso abordar en una próxima ocasión la situación de las publicaciones seriadas y periódicas, en particular las revistas, en todo lo relativo a su producción, registros ISSN, categorías temáticas, normas de edición y acreditación ante sistemas de difusión internacionales. 
Tabla A. Total de registros ISBN de las universidades chilenas. 2002-2004

\begin{tabular}{|c|c|c|c|c|}
\hline Nombre & 2002 & 2003 & 2004 & Total \\
\hline Pontificia Universidad Católica de Chile & 39 & 60 & 58 & 157 \\
\hline Universidad Católica del Norte & 29 & 42 & 30 & 101 \\
\hline Universidad de Chile & 31 & 34 & 30 & 95 \\
\hline Universidad Arturo Prat & 9 & 30 & 47 & 86 \\
\hline Universidad de Playa Ancha de Ciencias de la Educación & 25 & 27 & 22 & 74 \\
\hline Universidad de Concepción & 23 & 19 & 26 & 68 \\
\hline Universidad de Arte y Ciencias Sociales (ARCIS) & 29 & 10 & 8 & 47 \\
\hline Universidad Diego Portales & 6 & 26 & 15 & 47 \\
\hline Pontificia Universidad Católica de Valparaíso & 13 & 16 & 12 & 41 \\
\hline Universidad de Antofagasta & 3 & 19 & 16 & 38 \\
\hline Universidad de Los Lagos & 18 & 7 & 8 & 33 \\
\hline Universidad de Santiago & 4 & 12 & 16 & 32 \\
\hline Universidad Católica Silva Henríquez & 7 & 8 & 13 & 28 \\
\hline Universidad de la Frontera & 15 & 5 & 8 & 28 \\
\hline Universidad del Bío Bío & 7 & 7 & 4 & 18 \\
\hline Universidad de Valparaíso & 8 & 6 & 4 & 18 \\
\hline Universidad de Talca & 7 & 3 & 5 & 15 \\
\hline Universidad Bolivariana & 2 & 3 & 8 & 13 \\
\hline Universidad Metropolitana de Ciencias de la Educación & 1 & 5 & 5 & 11 \\
\hline Universidad de Magallanes & 2 & 3 & 5 & 10 \\
\hline Universidad de los Andes & 2 & 5 & 3 & 10 \\
\hline Universidad Andrés Bello & 7 & 1 & 2 & 10 \\
\hline Universidad Gabriela Mistral & 2 & 2 & 3 & 7 \\
\hline Universidad Mayor & 2 & 3 & 2 & 7 \\
\hline Universidad Academia de Humanismo Cristiano & 0 & 2 & 4 & 6 \\
\hline Universidad Católica del Maule & 3 & 2 & 1 & 6 \\
\hline Universidad José Santos Ossa & 2 & 2 & 2 & 6 \\
\hline Universidad Santo Tomás & 2 & 0 & 3 & 5 \\
\hline Universidad de Tarapacá & 2 & 2 & 1 & 5 \\
\hline Universidad Austral & 2 & 1 & 2 & 5 \\
\hline Universidad de Viña del Mar & 0 & 2 & 3 & 5 \\
\hline Universidad del Desarrollo & 0 & 2 & 2 & 4 \\
\hline Universidad San Sebastián & 1 & 1 & 1 & 3 \\
\hline Universidad Pérez Rosales & 0 & 1 & 2 & 3 \\
\hline Universidad de La Serena & 0 & 1 & 2 & 3 \\
\hline Universidad Adolfo Ibáñez & 1 & 1 & 0 & 2 \\
\hline Universidad La República & 2 & 0 & 0 & 2 \\
\hline Universidad Alberto Hurtado & 0 & 0 & 1 & 1 \\
\hline Universidad Iberoamericana de Ciencias y Tecnología & 1 & 0 & 0 & 1 \\
\hline Universidad de Ciencias de la Informática & 0 & 1 & 0 & 1 \\
\hline Universidad Mariano Egaña & 0 & 0 & 1 & 1 \\
\hline Total & 307 & 371 & 375 & 1053 \\
\hline
\end{tabular}

Fuente: Cámara Chilena del Libro. 